\title{
Changing Nomenclature for PBC from "Primary Biliary Cirrhosis" to "Primary Biliary Cholangitis"
}

\author{
Atsushi Tanaka $^{1,6} \cdot$ Hajime Takikawa ${ }^{1,6} \cdot$ Satoshi Mochida $^{4,8} \cdot$ Kazuhiko Koike $^{5,8}$. \\ Hiroto Miwa $^{2,7}$ - Toru Shimosegawa ${ }^{3,7}$
}

Received: 19 April 2016/Accepted: 19 April 2016/Published online: 26 May 2016

(C) Japanese Society of Gastroenterology 2016

Primary biliary cirrhosis (PBC) is a chronic cholestatic liver disease, frequently developing in middle-aged women [1]. In patients with $\mathrm{PBC}$, small bile ducts in the liver are believed to be damaged via autoimmune reactions [2]. Patients were diagnosed as having $\mathrm{PBC}$ when 2 or more among the following 3 features are observed; elevated cholestatic enzymes, presence of anti-mitochondrial autoantibodies (AMA) and histological findings as chronic non-suppurative destructive cholangitis (CNSDC) in

This article is published simultaneously in Hepatology Research, a title published by John Wiley and Sons. The Japanese version of this article is also published in Kanzo (The official Japanese journal of the Japan Society of Hepatology) and in Nihon Shokakibyo Gakkai Zasshi (The official Japanese journal of the Japanese Society of Gastroenterology). All versions of this article are published with full consent of both societies.

Atsushi Tanaka

a-tanaka@med.teikyo-u.ac.jp

1 Department of Medicine, Teikyo University School of Medicine, 2-11-1, Kaga, Itabashi-ku, Tokyo 173-8605, Japan

2 Division of Gastroenterology, Department of Internal Medicine, Hyogo College of Medicine, Hyogo, Japan

3 Division of Gastroenterology, Tohoku University Graduate School of Medicine, Sendai, Japan

4 Department of Gastroenterology and Hepatology, Faculty of Medicine, Saitama Medical University, Saitama, Japan

5 Department of Gastroenterology, Graduate School of Medicine, The University of Tokyo, Tokyo, Japan

6 Health Labor Science Research Grants from Research on Measures for Intractable Diseases, the Intractable HepatoBiliary Diseases Study Group in Japan, Tokyo, Japan

7 The Japanese Society of Gastroenterology, Tokyo, Japan

8 The Japan Society of Hepatology, Tokyo, Japan particular [3]. When the disease entity of PBC was established more than 50 years ago [4], advanced liver diseases showing histological findings of cirrhosis were found in the most of patients with PBC. Consequently, a nomenclature for primary biliary "cirrhosis" was reasonably accepted by all hepatologists and gastroenterologists world-widely.

Introduction of AMA as a tool for screening examination, however, enabled physicians to diagnose patients with chronic cholestatic diseases as having PBC during the earlier stages before developing cirrhosis [5]. Moreover, spread use of ursodeoxycholic acid (UDCA) as a first-line therapeutic agent, contributed to inhibit the progression of liver diseases from the non-cirrhotic stage to the cirrhotic stage in a large part of patients with PBC [6]. In Japan, about $70-80 \%$ of patients with $\mathrm{PBC}$ were shown to be diagnosed as having "asymptomatic PBC" in the recent years [3]. Thus, the nomenclature of primary biliary "cirrhosis" is regarded as a misnomer both from clinical and histological aspects. As a result, the patients inevitably misunderstand the stage of their diseases and at the same time have to bear the substantial burden in their social activities.

During 2014 and 2015, the governing boards of both the European Association of the Study for the Liver (EASL) and American Association of the Study for the Liver (AASLD) have approved the change of nomenclature for PBC from "primary biliary cirrhosis" to "primary biliary cholangitis", and published the statements in the official journals $[7,8]$, followed by publishing identical articles in several major journals in the fields of gastroenterology and Hepatology [9-11]. In contrast, in Japan, the Intractable Hepato-Biliary Diseases Study Group supported by Health Labor Science Research Grants from Research on Measures for Intractable Diseases performed the questionnaire survey regarding the nomenclature for PBC on 18 
experts for clinical research of PBC in 2014. Although all the experts agreed to revise the nomenclature for PBC, unanimous consent was not obtained. Of the 18 Japanese experts, 11 supported the nomenclature of "cholangitis", while 5 and 2 experts proposed "cholangiopathy" and the other nomenclatures, respectively. "Biliary" and "cholangitis" may sound as redundant repetition, and "cholangitis" does not reflect pathological features observed in the liver of patients with PBC exactly. Considering consistency with the nomenclature approved by EASL and AASLD, the Study Group decided to adopt the nomenclature of "primary biliary cholangitis" to keep a well-known abbreviation "PBC". However, an alternative nomenclature for $\mathrm{PBC}$, which more accurately represents the disease entity, should be established in the future.

In December 2015, the Intractable Hepato-Biliary Diseases Study Group of Japan addressed the proposal regarding revision of nomenclature for $\mathrm{PBC}$ to the governing boards of the Japanese Society of Gastroenterology (JSGE) and the Japan Society of Hepatology (JSH), and the nomenclature of "primary biliary cholangitis (PBC)" was approved in January 2015 and March 2015 by JSH and JSGE, respectively. Both societies proposed the novel nomenclature jointly to the Japanese Association of Medical Sciences (JAMS) in April 2014, and the nomenclature of "primary biliary cholangitis", will be officially used by the Ministry of Health, Labor and Welfare of Japan following discussion by the committee for regulation of medical terms organized in JAMS.

We sincerely call on all members of the Japanese Society of Gastroenterology and the Japan Society of Hepatology to use from this moment on the name "primary biliary cholangitis", instead of primary biliary "cirrhosis", for the disease known by its abbreviation PBC.

\section{Compliance with ethical standards}

Conflict of interest The authors declare that they have no conflict of interest.

\section{References}

1. Carey EJ, Ali AH, Lindor KD. Primary biliary cirrhosis. Lancet. 2015; 17; 386(10003):1565-75.

2. Gershwin ME, Ansari A, Mackay I, et al. Primary biliary cirrhosis: an orchestrated immune response against epithelial cells. Immunol Rev. 2000;174:210-25.

3. Working Subgroup (English version) for Clinical Practice Guidelines for Primary Biliary Cirrhosis. Guidelines for the management of primary biliary cirrhosis: the intractable hepatobiliary disease study group supported by the Ministry of health, labour and welfare of Japan. Hepatol Res. 2014;44 (Suppl S1):71-90.

4. Dauphinee JA, Sinclair JC. Primary biliary cirrhosis. Can Med Assoc J. 1949;61:1-6.

5. Gershwin ME, Mackay I, Sturgess A, et al. Identification and specificity of a cDNA encoding the $70 \mathrm{kd}$ mitochondrial antigen recognized in primary biliary cirrhosis. J Immunol. 1987;138: $3525-31$.

6. Abbas G, Lindor KD. Pharmacological treatment of biliary cirrhosis with ursodeoxycholic acid. Expert Opin Pharmacother. 2010;11:387-92.

7. Beuers U, Gershwin ME, Gish RG, et al. Changing nomenclature for PBC: From 'cirrhosis' to 'cholangitis'. J Hepatol. 2015;63:1285-7.

8. Beuers U, Gershwin ME, Gish RG, et al. Changing nomenclature for PBC: From 'cirrhosis' to 'cholangitis'. Hepatology. 2015;62:1620-2.

9. Beuers U, Gershwin ME, Gish RG, et al. Changing Nomenclature for PBC: From 'Cirrhosis' to 'Cholangitis'. Gastroenterology. 2015;149:1627-9.

10. Beuers U, Gershwin ME, Gish RG, et al. Changing nomenclature for PBC: from 'cirrhosis' to 'cholangitis'. Gut. 2015;64:1671-2.

11. Beuers U, Gershwin ME, Gish RG, et al. Changing Nomenclature for PBC: From 'Cirrhosis' to 'Cholangitis'. Am J Gastroenterol. 2015;110:1536-8. 